\title{
La perception des inégalités et la promotion de la justice sociale par de futur-es enseignant-es issu-es de la migration
}

\author{
Olivier Delévaux, Haute École Pédagogique du Canton de Vaud
}

DOI : 10.51186/journals/ed.2021.11-1.e430

\section{Résumé}

Les constats liés à la surreprésentation des élèves issu-es de la migration dans les filières de formation à exigences élémentaires ou dans les structures de l'enseignement spécialisé montrent que, malgré la mise en évidence depuis de nombreuses années des mécanismes de discrimination systémique opérant au sein du système scolaire, l'évolution en termes de réussite éducative n'est pas satisfaisante. Notre recherche qualitative s'appuie sur 25 entretiens semi-dirigés réalisés auprès de futur-es enseignant-es primaires issu-es de la migration de la Haute école pédagogique du canton de Vaud à Lausanne. Elle vise à mettre en évidence des expériences d'injustices dans leur propre parcours, mais également leurs observations de discriminations liées à l'origine des élèves dans le cadre des stages et à évoquer des pistes visant un renforcement de la justice sociale au sein de l'école. Les résultats montrent que la quasi-totalité des personnes interrogées a été confrontée personnellement à des difficultés en lien avec leur histoire migratoire. Leurs parcours scolaires et de formation sont souvent parsemés d'embûches, peu linéaires, et parfois marqués par des injustices manifestes. Ils sont également souvent témoins, lors de leurs stages, de manifestations de discriminations visant les élèves issu-es de la migration. Les pistes évoquées impliquent les enseignant-es et s'orientent vers les élèves, mais surtout vers leurs familles.

Mots-clés : discrimination systémique, futur-es enseignant-es, inégalités scolaires, issu-es de la migration, justice sociale

\section{Abstract}

Mechanisms of systemic discrimination operating within the school system have been highlighted for many years. Despite this fact, research has shown an over-representation of students with a migrant background in special education structures. This observation demonstrates that the evolution in terms of educational equity is not satisfactory. Our qualitative research is based on 25 semi-structured interviews with future primary school teachers with a migration background at Lausanne University of Teacher Education. It aims to 
highlight experiences of injustice in their own careers, but also their observations of discrimination related to the origin of students in the context of internships. Moreover, we wanted to explore possible ways to strengthen social justice within the school. Our results show that almost all the interviewees were personally confronted with difficulties related to their migration history. Their educational and training paths are often strewn with pitfalls, not very linear, and sometimes marked by obvious injustices. During their internships, they also often witnessed manifestations of discrimination against students with a migration background. The possible solutions mentioned involve the teachers and are directed towards the students themselves but especially towards their families.

Keywords: educational inequalities, future teachers, migrant backgrounds, social justice, systemic discrimination

\section{INTRODUCTION}

La population issue de la migration constitue une part importante de la population résidente suisse. À titre indicatif, elle représente actuellement, selon l'Office fédéral de la statistique, $36 \%$ de la population résidante permanente du pays. Le parcours des jeunes issu-es de la migration ${ }^{1}$ dans le système éducatif constitue à nos yeux un enjeu majeur en termes de participation de cette population au sein de la société. Dans le canton de Vaud où se situe notre recherche, si le pourcentage de ces élèves au sein de la scolarité obligatoire est globalement comparable aux chiffres concernant l'ensemble de la population, le taux d'enseignant-es de nationalité étrangère au primaire (1ère à 8ème année de scolarité obligatoire) et au secondaire I (9, 10 et 11ème année de scolarité obligatoire) est cependant beaucoup plus faible (8,2\% pour l'ensemble de la scolarité obligatoire $\left.{ }^{23}\right)$. On pourrait y voir, entre autres explications, une conséquence des mécanismes qui aboutissent à une sousreprésentation des jeunes issu-es de la migration dans les filières de formation à exigences élevées. La thèse de doctorat en cours dont est issu cet article se focalise sur le parcours biographique et de formation de futur-es enseignant-es issu-es de la migration. Nous cherchons en particulier à mettre en évidence les facteurs qui, en dépit du fait qu'elles/ils ont été confronté-es à des obstacles ou à des manifestations de discriminations systémiques (Dhume, 2016), leur ont permis d'accéder à l'enseignement supérieur et les ont conduit-es au choix d'une formation à l'enseignement. Nous considérons également qu'en raison de leurs expériences spécifiques, elles/ils constituent des observateurs et observatrices privilégié-es de la scolarité des élèves issu-es de la migration qu'elles/ils rencontrent à

\footnotetext{
1 Nous utilisons le terme migration, au singulier, pour désigner un processus général de déplacement caractérisant la biographie familiale des personnes concernées par notre recherche.

2 Source : Office fédéral de la statistique : Personnel des écoles 2019/20: scolarité obligatoire, degré secondaire II et degré tertiaire (écoles supérieures) https://www.bfs.admin.ch/bfs/fr/home/statistiques/education-science/ personnel-institutions-formation.assetdetail.16444025.html

3 Ce taux serait différent si les statistiques tenaient compte des binationalités.
} 
l'occasion de leurs stages, et qu'elles/ils sont à même d'évoquer des pistes pertinentes pour favoriser leurs réussites scolaire et éducative.

Dans un premier temps, nous situerons l'objet de la recherche en regard d'éléments venant alimenter la réflexion sur les inégalités scolaires et sociales. Nous proposerons ensuite une réflexion sur les notions d'égalité et d'inégalités scolaires (Dubet, 2009), et une tentative de précision des dimensions de la notion de justice sociale dans le contexte qui nous intéresse (Forsé, 2014 ; Fraser, 2005). Finalement, après quelques précisions méthodologiques, nous exposerons et discuterons nos résultats intermédiaires et tenterons de mettre en évidence les liens entre les données recueillies et des dimensions retenues de la justice sociale, dans un but d'éclairer les mécanismes à l'œuvre et de promouvoir des mécanismes favorisant la résilience et la réussite éducative.

\section{SCOLARITÉ DES ÉLÈVES ISSU-ES DE LA MIGRATION}

II y a presque vingt ans, dans la foulée des résultats de l'enquête PISA 2000, un rapport mettait en évidence la surreprésentation des élèves issu-es de la migration dans les voies de formation à exigences élémentaires (Conférence suisse des directeurs cantonaux de l'instruction publique, 2003). Kronig (2003), par exemple, relevait que :

Non seulement la part des enfants immigrés dans la catégorie des élèves à faible capacité d'apprentissage est disproportionnée, mais elle est en croissance continue. Leur nombre n'a augmenté que d'un tiers dans les classes ordinaires tandis qu'il a triplé dans les classes spéciales. [...] Ces développements sont comparables au processus de la création de sous-couches sur le marché de l'emploi, tel qu'il a été décrit par les sociologues au cours des années 70. (p. 24)

L'auteur rappelait également que les premières recommandations de la CDIP $^{4}$ concernant une meilleure prise en compte de la diversité culturelle des élèves avaient été formulées au début des années 1970, mais qu'elles étaient restées sans effet sur le nombre d'assignations des élèves immigré-es aux structures de l'enseignement spécialisé. Pour lui, cet état de fait est à mettre en lien avec une conception réductrice de l'échec scolaire des élèves issu-es de la migration qui ne tient pas compte d'autres variables que les difficultés individuelles d'apprentissage.

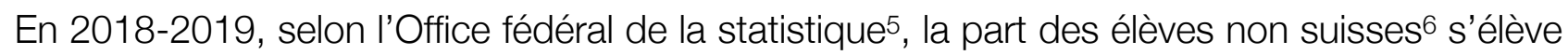
à $28 \%$ pour l'école obligatoire en moyenne nationale, ce taux variant cependant fortement d'un canton à l'autre. Ce pourcentage est en constante évolution. À titre de comparaison, la

\footnotetext{
${ }^{4}$ Conférence suisse des directeurs cantonaux de l'instruction publique

5 Sources pour l'ensemble des pourcentages présentés : https://www.bfs.admin.ch/bfs/fr/home/statistiques/ education-science/personnes-formation/ecole-obligatoire.html

${ }^{6}$ La statistique s'appuie sur la nationalité des élèves scolarisé-es et non sur le statut migratoire et fait ainsi mention « d'élèves étrangers »).
} 
moyenne nationale était d'un peu moins de $22 \%$ en $2000-2001$. Nous nous intéresserons particulièrement à la sélection en fin d'école primaire et à l'orientation vers des voies d'étude différenciées par leur niveau d'exigence au secondaire I. En 2018-2019, l'école primaire, organisée en tronc commun, montre un pourcentage d'élèves d'origine étrangère proche des $28 \%$ évoqués pour l'école obligatoire dans son ensemble, tout comme le secondaire I considéré dans son ensemble. Lorsque l'on considère les voies du secondaire I à exigences élémentaires, on constate cependant que la part d'élèves d'origine étrangère s'élève à plus de $37 \%$, alors qu'elle est d'un peu moins de 18\% dans les voies du secondaire I à exigences étendues. Ce constat vient étayer les témoignages des étudiant-es issu-es de la migration que nous avons interrogé-es qui, souvent, font état d'un constat de minorisation lors de leur orientation vers des voies pré-gymnasiales ${ }^{7}$ et, plus tard, lors de leur accès à une école de maturité gymnasiale qui leur ouvrira les portes de l'enseignement supérieur.

Concernant l'enseignement spécialisé, nous constatons des variations importantes du pourcentage d'élèves d'origine étrangère selon le type d'offre de l'enseignement spécialisé. En moyenne suisse, ces élèves représentent près de $27 \%$ de l'effectif des élèves bénéficiant de mesures de l'enseignement spécialisé en classe ordinaire, soit un chiffre proche du taux relevé pour l'école obligatoire dans son ensemble. Ils sont près de $56 \%$ dans les classes spéciales des établissements scolaires et environ 37\% dans les écoles spécialisées. Ceci met en évidence que, si le taux d'élèves d'origine étrangère bénéficiant d'une offre de pédagogie spécialisée au sein de l'école ordinaire est comparable au taux de leur présence dans l'école obligatoire, il est beaucoup plus important lorsque les mesures sont ségrégatives.

\section{DES FUTUR-ES ENSEIGNANT-ES ISSU-ES DE LA MIGRATION}

Les constats précédemment identifiés sont les mêmes dans les pays voisins, par exemple en France (Brinbaum, et al., 2012) ou en Allemagne (Allemann-Ghionda, et al., 2006 ; Gomolla \& Radtke, 2009), et les facteurs de risque identifiés (Coradi Vellacott \& Wolter, 2002) sont globalement les mêmes (p. ex. attentes inférieures de l'école face à l'élève, en lien avec la situation familiale, influence du capital économique et culturel, allophonie).

Plutôt que de nous focaliser sur les facteurs de risques, nous avons choisi de nous intéresser aux facteurs de protection, et pour ce faire, d'analyser les parcours biographiques et de formation de futur-es enseignant-es issu-es de la migration. L'augmentation régulière de leur nombre au sein des cohortes d'étudiant-es nous a interpellé et nous a amené à vouloir en savoir plus sur leur parcours, leurs motivations, leurs perceptions de la justice sociale au sein du système éducatif. Nous avons pu, au travers de nos contacts avec elles et eux, prendre

\footnotetext{
7 En Suisse, le secondaire 2, qui fait suite à la scolarité obligatoire, propose deux voies principales : voie maturité gymnasiale qui donne accès aux hautes écoles et aux universités, et voie formation professionnelle initiale qui débouche sur un certificat fédéral de capacité. Le secondaire 1 introduit généralement une sélection qui pré-oriente des élèves vers l'une des deux voies.
} 
conscience de la diversité et de la complexité des trajectoires qui les ont amené-es jusqu'à une formation d'enseignant-es.

Notons qu'il n'y a pas, du moins en Suisse romande, de politique active de recrutement ou de formation d'enseignant-es issu-es de la migration telle qu'on peut en trouver en GrandeBretagne (Charles \& Legendre, 2006) ou en Allemagne (Allemann-Ghionda, et al., 2006). ॥ s'agit dans cette perspective de favoriser l'engagement d'enseignant-es formé-es à l'étranger ou d'encourager la formation chez les jeunes issu-es de la migration, en escomptant chez elles et eux des compétences particulières en matière de prise en compte de la diversité culturelle des élèves. De fait, l'accès à la formation à l'enseignement pour des personnes d'origine étrangère, notamment pour le degré primaire, ne s'est ouvert dans notre pays qu'avec l'apparition de hautes écoles pédagogiques et la délivrance d'un titre reconnu au niveau national. De manière générale, le recrutement du personnel enseignant n'est pas soumis non plus à des concours, mais se fait sur la base de postulations libres. Par conséquent, l'intérêt pour ces formations auprès des jeunes issu-es de la migration est un phénomène assez récent, qui, même s'il est en augmentation régulière, reste encore assez marginal. Selon notre observation, basée sur les statistiques internes de la Haute école pédagogique du canton de Vaud et les entretiens réalisés, cet intérêt concerne surtout des personnes issues de la migration de deuxième génération, nées en Suisse ou y ayant effectué la plus grande partie de leur scolarité.

Au travers de notre recherche, nous souhaitons tout particulièrement identifier ce qui leur a permis d'accomplir un parcours caractérisé par la réussite scolaire et connaître ce qui, de leur point de vue de témoins privilégié-es, serait à même de réduire les inégalités et donc de renforcer la justice sociale au sein du système éducatif.

\section{INÉGALITÉS ET JUSTICE SOCIALE}

Nous empruntons à Dubet (2009) une classification des inégalités scolaires en trois types : égalité d'accès, égalité des chances et égalité des résultats.

La première peut être assimilée à la distribution des biens scolaires à l'instar d'autres biens de consommation. On pourrait considérer a priori qu'en Suisse, comme dans les pays qui l'entourent, cette forme d'égalité ne constitue plus un enjeu, du moins pour la formation élémentaire.

L'égalité des chances prend son sens lorsque l'égalité d'accès est garantie : " Ce modèle domine aujourd'hui dans les sociétés qui considèrent que tous les individus sont fondamentalement égaux et qu'ils doivent donc occuper des positions sociales inégales en fonction de leur seul mérite »(Dubet, 2009, p. 19). Ce modèle est donc étroitement lié à l'idée de méritocratie qui justifie sélection scolaire et orientation vers des filières différenciées en termes d'exigences, mais également de débouchés professionnels. Sous couvert de 
principe d'égalité, on admet donc l'existence d'inégalités si l'on considère l'accessibilité à l'ensemble des offres de formation et des perspectives professionnelles.

L'égalité des résultats, quant à elle, tente de corriger l'approche méritocratique. Dans cette perspective, le système scolaire le plus juste vise à diminuer les inégalités entre les moins bons et les meilleurs élèves, ou encore à rapprocher les résultats des enfants d'ouvrières/ers de ceux des enfants de cadres (Dubet, 2009).

On considère ainsi prioritairement les élèves les plus vulnérables, et on alloue plus de moyens à la formation élémentaire, privilégiant les troncs communs et la mixité sociale. Poussée plus loin, cette logique peut aboutir à la mise en place de mesures de discrimination positive et introduire ainsi certaines formes d'inégalités considérées comme justes. L'égalité des résultats peine à se développer dans notre système éducatif, et le constat que nous faisions plus haut quant à la surreprésentation des élèves issu-es de la migration dans les filières de formation à exigences élémentaires illustre bien la conviction méritocratique qui anime les politiques scolaires, et qui se manifeste notamment par les craintes souvent exprimées de baisse des exigences liées à l'application de principes d'égalité des résultats.

Les élèves les plus vulnérables, dont les élèves issu-es de la migration font souvent partie bien que nous refusions tout amalgame, sont donc confronté-es à un système qui pratique une sélection précoce et qui les oriente vers des filières différenciées. Cette orientation se fonde non seulement sur leurs performances scolaires, mais également parfois sur des préjugés et stéréotypes quant à leurs capacités futures à évoluer dans une filière à exigences élevées, ou quant aux compétences supposées de leur famille à fournir un accompagnement adéquat dans leur scolarité. Meyer (2009), mentionnant la recherche de Kronig (2007) sur l'exactitude de la mesure, l'équité de l'évaluation et sa qualité pronostique, relève à ce sujet que la " décision de sélection dépend également fortement du contexte de la classe, du corps enseignant qui procède à l'évaluation et surtout de caractéristiques sans rapport avec les performances, telles que le sexe, la couche sociale ou l'origine migratoire des élèves testés » (p. 69).

Les concepts de justice sociale et de justice distributive contribuent à éclairer la question des égalités scolaires ou à établir en quoi certaines inégalités peuvent être considérées comme justes. Michel Forsé (2014) relève trois critères de justice sociale :

Le premier vise à satisfaire les besoins (au moins) élémentaires de chacun. Le deuxième s'appuie sur l'équité, c'est-à-dire une rétribution proportionnelle aux investissements des uns et des autres dans ce qui se trouve être collectivement à distribuer. Concrètement, il s'agit le plus souvent de faire en sorte que chacun soit rétribué selon ses mérites propres [...]. Le troisième principe cherche à obtenir une stricte égalité de chacune des parts. (p. 108) 
On voit bien la superposition possible de ces critères avec les formes d'égalité proposées par Dubet (2009). Forsé (2014) attire l'attention sur leur incompatibilité entre eux a priori, et sur la nécessité de les hiérarchiser. L'auteur, citant Deutsch (1975; 1985), mentionne que:

Dans les situations où l'objectif premier de la coopération est la productivité économique, le mérite [le critère d'équité] est le critère dominant de justice distributive. Dans les situations où l'objectif premier de l'interaction humaine est la recherche ou l'entretien de relations sociales pour elles-mêmes, l'égalité est le critère dominant. Enfin, dans les situations où l'objectif premier de la coopération est le développement personnel et le bien-être personnel, le besoin est le critère dominant. (Forsé, 2014, p. 114)

Les résultats de l'enquête European Values Study (EVS) réalisée à l'échelle européenne en 1999 montrent un consensus quant à la hiérarchisation des critères :

Parmi les critères de justice, s'il faut n'en retenir qu'un, ce sera avant tout la garantie des besoins de base. S'il faut en retenir deux, ce seront en priorité cette garantie associée à la reconnaissance des mérites. Quant à la réduction des inégalités, elle représente, comme nous l'avons vu, le critère le moins cité relativement aux deux autres, mais lorsqu'elle est jugée importante, c'est très souvent sous la condition que la reconnaissance des mérites ait déjà été jugée telle. (Forsé, 2014, p. 118)

Ceci vient confirmer notre sentiment quant aux obstacles qui se dressent contre l'application d'une forme d'égalité scolaire recourant moins aux principes méritocratiques. Si la satisfaction des besoins élémentaires ne fait pas débat, elle ne constitue cependant plus un enjeu premier en Suisse. L'école est par contre soumise, au sein d'une société très libérale, à des tensions entre les convictions productivistes qui privilégient une distribution des biens (et des orientations scolaires) basée sur le mérite et des principes prônant une réduction des inégalités par l'égalité des résultats ou, plus loin, l'égalité d'accès à l'emploi.

De manière complémentaire à ces éléments, nous trouvons particulièrement intéressante l'approche de Fraser (2005) pour qui « la justice implique à la fois la redistribution et la reconnaissance » (p. 13). Pour l'autrice, alors que la justice redistributive s'attacherait à corriger les injustices de type socioéconomique, la justice liée à la reconnaissance s'appliquerait davantage aux situations d'injustice de type culturel. Fraser (2005) met cependant également en évidence le fait que les deux types sont souvent liés et que cette approche comporte des limites et présente un dilemme : " les personnes qui sont objets simultanément d'injustice culturelle et d'injustice économique ont besoin à la fois de reconnaissance et de redistribution ; elles ont besoin à la fois de revendiquer et de nier leur spécificité » (2005, p. 22). Elle ajoute à son modèle une dimension supplémentaire transversale à l'axe "redistribution/reconnaissance » et propose deux manières de « remédier » à l'injustice qu'elle appelle respectivement « remèdes correctifs » et « remèdes transformateurs » : 
Les remèdes correctifs à l'injustice sont ceux qui visent à corriger les résultats inéquitables de l'organisation sociale sans toucher à leurs causes profondes. Les remèdes transformateurs, pour leur part, visent les causes profondes. L'opposition se situe entre symptômes et causes, et non entre changement graduel et transformation radicale. (Fraser, 2005, pp. 34-35)

L'auteure propose donc un modèle qui combine en quatre quadrants types de justice et remèdes. Sa proposition est de privilégier les remèdes transformateurs, qu'ils soient appliqués à la justice redistributive ou à la reconnaissance, plus à même d'apporter des modifications sociétales profondes.

Confronté-es à une école dans laquelle la méritocratie prédomine et donc exposés à des facteurs de risque, certain-es élèves a priori vulnérables parviennent tout de même à développer de la résilience et à accomplir, même si ce n'est pas sans difficulté, un parcours de formation en lien avec leurs potentialités. Notre objectif est de rendre visibles les obstacles rencontrés et observés, mais aussi ce qui apparaît comme facteurs de protection dans le parcours biographique et de formation de futur-es enseignant-es issu-es de la migration. Nous souhaitons également mettre en évidence ce qui leur paraît constituer des pistes d'amélioration de la justice sociale dans l'école en favorisant une meilleure réussite scolaire et éducative des élèves issu-es de la migration. Nous tenterons de mettre en relation ces constats et propositions avec les critères de justice sociale évoqués plus haut.

\section{MÉTHODOLOGIE}

Dans une logique de recherche qualitative, et à partir d'une démarche descriptive et interprétative, la visée de la recherche sur laquelle s'appuie cet article est de permettre une meilleure compréhension des phénomènes à l'œuvre (Karsenti \& Savoie-Zajc, 2011) pour favoriser la réussite des parcours de formation d'élèves issu-es de la migration.

Nous nous appuyons ici sur les données recueillies au travers d'une première série de 25 entretiens semi-dirigés (Kaufmann, 2011 ; Savoie-Zajc, 2009) avec des étudiant-es en dernière année de formation en enseignement primaire. Notre choix s'est porté sur cet échantillon pour deux raisons principales : concernant l'enseignement primaire, il s'agit des futur-es enseignant-es qui sont et seront le plus confronté-es à l'hétérogénéité des classes puisqu'intervenant dans le tronc commun de la scolarité. Nous avons ensuite privilégié des étudiant-es en fin de formation d'une part parce qu'elles/ils ont un bagage expérientiel lié au stage un peu plus conséquent, mais également, car nous avons pour projet de reprendre contact après une année d'expérience professionnelle, soit l'année suivant le premier entretien, afin de confronter certaines données à leur pratique professionnelle. 
Le recrutement des participant-es s'est fait via un courriel, adressé à l'ensemble des étudiant-es de la cohorte, qui présentait la recherche et ses buts, ainsi que le profil des étudiants recherchés. Les 25 étudiant-es correspondant au profil défini (étudiant-es pour l'enseignement primaire né-es en Suisse de parents migrants ou ayant elles-mêmes ou euxmêmes migré et accompli une partie de leur scolarité en Suisse) et intéressé-es à contribuer à la recherche se sont ensuite manifesté-es par retour de courriel. L'accès aux étudiant-es a été facilité par l'appartenance de l'auteur-e à l'institution de formation.

Les entretiens ont fait l'objet d'une transcription puis d'un traitement qualitatif par codage et catégorisation (Van der Maren, 1996) réalisé à l'aide du logiciel NVivo 12.

\section{RÉSULTATS}

\subsection{Parcours non linéaires et discriminations}

Les entretiens réalisés ont révélé une grande diversité de parcours biographiques et de formation, nous renforçant dans la conviction qu'il est nécessaire de considérer les individualités et l'unicité des histoires en premier lieu. Même si certaines tendances se dégagent parmi les 25 étudiant-es interrogé-es ici, les raisons de la migration, le statut migratoire, le statut socioculturel de la famille, la langue maternelle, le nombre d'années de scolarité effectuées en Suisse, pour ne citer que ces éléments, sont autant de variables qui influencent la diversité de leurs parcours. Parmi les points communs relevés, dans leur très grande majorité, les cursus de formation n'ont pas été linéaires, et la quasi-totalité des personnes interrogées relatent avoir été confrontées à de la discrimination durant leur scolarité.

Dans plusieurs cas, des étudiant-es nous ont rapporté n'avoir pas perçu, sur le moment, que certains actes ou propos étaient discriminatoires, mais en avoir pris conscience plus tard. L'âge au moment des faits est parfois évoqué, ainsi que l'impression d'une certaine naïveté poussant à considérer que ce qui se passait était «normal »: "Plus on grandit, plus on comprend de choses et plus on se rend compte qu'il y a des formes de... je ne dirais pas racisme, mais en tout cas de rabaissement » ( $\mathrm{v}$., 24 ans).

Cette discrimination peut prendre des formes diverses et nous nous concentrons ici sur des exemples qui ont un lien direct avec la scolarité. Plusieurs étudiant-es font part de difficultés présupposées ou anticipées qui se sont avérées sans fondement :

On m'a dit : " Ah, mais tu devras travailler beaucoup plus, tu auras des difficultés en français, tu auras des difficultés en maths ». Et au final, quand je l'ai réussi, je me suis dit : "Mais de quelles difficultés ils parlaient ? ». (M., 22 ans) 
L'anticipation de difficultés scolaires est parfois justifiée par les professionnel-les par le fait que la langue parlée à la maison est différente de la langue de scolarisation et/ou par la conviction que les parents ne pourront pas accompagner la scolarité de leur enfant.

Parfois, il s'agit de mise en doute des capacités qui semblent ne reposer que sur des préjugés liés à l'origine migratoire des élèves :

Le dernier jour [d'école obligatoire], malgré mes excellentes notes et les certificats que j'avais, la maîtresse de classe n'a pas voulu me donner mon... vous savez, on appelle ça le « carnet blanc » en fait, à l'intérieur il y a le résumé des trois ans avec tous les certificats, toutes les notes. On peut le prendre si on veut par exemple aller au gymnase ${ }^{8}$ et si on ne veut pas forcément le faire, ou si on veut faire un apprentissage ou autre, ils peuvent le garder eux. Et je me souviens que j'avais dit que moi j'en ai besoin, moi je vais au gymnase, «non, mais tu es sûre que tu en as besoin ? ». (F., 24 ans)

Certain-es mentionnent également des situations ouvertement xénophobes : " Vous, vous n'allez jamais réussir'. Et elle le disait sans aucune gêne à tous les étrangers » (M., 23 ans).

\subsection{Sélection et orientation : une autre forme d'inégalité d'accès}

Un certain nombre de ces manifestations discriminatoires est directement lié au processus de sélection et d'orientation en fin d'école primaire, lui-même en cause dans les parcours non linéaires que nous avons relevés.

Les futur-es enseignant-es que nous avons rencontré-es ont pour la plupart connu l'ancien système de sélection de l'école vaudoise qui opérait, en fin de 6ème année primaire de l'époque (8H maintenant), une orientation vers trois voies possibles constituant le troisième cycle de formation obligatoire (secondaire l) et correspondant à une gradation des exigences : voie secondaire à options (VSO), voie secondaire générale (VSG) et voie secondaire de baccalauréat (VSB). L'accès aux différents types de formations postobligatoires est largement conditionné par cette sélection. Des possibilités de raccordement entre les voies sont cependant proposées en cours de formation secondaire I. Cinq personnes interrogées sur les 25 y ont eu recours.

L'enjeu de cette sélection est donc important, et les témoignages recueillis à ce sujet sont parfois éloquents en termes de discriminations et de mise à mal des critères d'égalité scolaire. Certain-es font état de doutes clairement évoqués quant aux capacités de la personne de poursuivre sa scolarité dans une voie à exigences étendues :

Je me souviens quand je devais aller en VSB, j'avais donc toutes mes notes, tous les quotas que j'avais à respecter, toutes les demandes, les contraintes etc. Mais

\footnotetext{
8 Dans certains cantons suisses, les gymnases sont les établissements post-obligatoires qui délivrent la maturité gymnasiale.
} 
l'enseignante a quand même voulu me diriger dans une voie plus « basse » donc la voie générale, plus par raison... on va dire parce que j'étais étrangère. (D., 23 ans)

Nous avons également constaté que cela pouvait être le cas lors d'une arrivée en fin de scolarité en Suisse, par la non-prise en compte de la scolarité précédente et une orientation vers la voie de formation à exigences les plus réduites avec les risques que cela implique en termes de perspectives de formation professionnelle ultérieure, ou les conséquences, comme c'est le cas ici, en termes de rallongement des études afin de réaliser les différentes passerelles jusqu'à l'accès à l'enseignement universitaire :

Je pense qu'il a fallu trouver une solution, me recaser. Donc j'ai fait 9ème terminale VSO. [...] Mais je n'arrive pas définir sur quoi mon orientation a été faite. Donc non, parce que je le gymnase, je l'ai trouvé abordable. (R., 35 ans, arrivée à 15 ans en Suisse)

D'autres témoignent d'injustices clairement constatées, les mêmes choix n'étant pas offerts à des élèves dans des situations comparables :

Une de mes camarades avait 14 points et demi. Le prof a dit " tu seras en VSG » alors qu'une fille qui était [...] Suisse, et qui avait aussi 14 points et demi a eu le choix entre VSB et VSG. (S., 21 ans)

Nous pourrions nous demander si, dans ces cas de figure, l'égalité d'accès à une partie de la scolarité obligatoire, semblant pourtant garantie par le système scolaire, n'est pas compromise. Les données recueillies, nous l'avons relevé, correspondent à un système de sélection qui n'est plus en vigueur. Une sélection en fin de 8ème année de scolarité obligatoire est cependant toujours présente et, de notre point de vue, n'amène pas de réel changement quant aux risques évoqués.

\subsection{Importance des enseignant-es}

De manière générale, l'attitude de certain-es enseignant-es peut exercer une influence, négative ou positive, sur le sentiment de compétence personnelle des étudiant-es interrogées et sur leur orientation. Pour certaines personnes, le regard bienveillant ou les encouragements d'un-e enseignant-e se sont avérés déterminants :

J'avais une prof qui m'avait laissé (je l'ai toujours) un petit mot qui me disait « écoute c'est très bien ton truc d'apprentissage, tu en es capable, mais moi je pense réellement que tu es capable aussi pour faire des études. Donc réfléchis bien, mais moi je te verrais bien au gymnase. (C., 21 ans)

Par ailleurs, l'importance d'une rencontre avec un-e enseignant-e qui les a positivement marqué-es est presque toujours mise en lien avec le choix de devenir à leur tour enseignante. Ce constat peut être rapproché des travaux de Villegas et Irvine (2010) et de Bauer et Akkari (2016) mettant en évidence la notion de role model. C. décrit l'importance de sa 
présence et de ses discours sur son parcours. II est intéressant de relever que cette rencontre, qui a motivé à viser des voies de formation à exigence élevées ou à y persévérer, ne concerne pas seulement des enseignant-es de la scolarité obligatoire, mais également, assez souvent, des enseignant-es du secondaire II. Ces dernières/ers se sont donc révélé-es important-es non pas pour l'orientation ou pour un changement de voie de formation, mais plutôt à un moment où la persévérance dans le projet d'études était mise à mal, en lien notamment avec le sentiment de minorisation ou de ne pas se sentir à sa place.

\subsection{Facteurs externes à l'école}

Certains facteurs extérieurs à l'institution scolaire sont aussi révélateurs et influent sur les choix d'orientation. Ainsi les questions de loyauté au groupe de pairs, la peur d'être marginalisé-es en optant, en cas de choix possible, pour une voie de formation à exigences élevées synonyme de minorisation pour des élèves issu-es de la migration, sont mentionnées par plusieurs étudiant-es : " Je ne voulais pas aller en VSB, je voulais aller en VSG pour être avec mes amis et tout ça. [...] Moi je préférais être avec des gens issus de la migration » (C., 21 ans).

Considérant que toutes les personnes interrogées fréquentent l'enseignement universitaire, ces préférences pour des voies ne préparant a priori pas à ce parcours ont eu une incidence sur la longueur des cursus d'études passant par diverses possibilités de passerelles.

Nous avons cependant aussi noté que dans certains cas, des attentes très élevées des parents quant à la réussite scolaire de leurs enfants, ainsi que certaines représentations quant à cette réussite (par exemple : études universitaires en médecine ou en économie) avaient pu jouer un rôle protecteur au cours de la scolarité obligatoire et de l'orientation dans des filières à exigences élevées. Elles ont cependant pu également inciter les jeunes à se diriger vers des voies d'études universitaires qui correspondaient davantage à l'aspiration de leurs parents qu'à leurs propres souhaits, ce qui s'est traduit après quelques semestres par une réorientation vers la formation à l'enseignement.

Les obstacles qui ont contribué à la non-linéarité des parcours ne sont donc pas exclusivement liés à l'institution scolaire, mais également à des facteurs personnels et familiaux. II convient également de relever que, si des facteurs de risque ont été identifiés, il existe aussi, dans les différentes sphères concernées, des facteurs de protection, notamment des personnes, qui ont joué un rôle favorable à la résilience lors de confrontation à des difficultés, ou à l'encouragement à poursuivre la formation. II n'en demeure pas moins que le regard porté sur certain-es élèves parce qu'elles/ils sont issu-es de la migration semble jouer un rôle particulier, indépendamment par exemple de leur lieu d'habitation ou de leur statut socioéconomique ou socioculturel. II est frappant de constater que la majorité des personnes interrogées, bien qu'ayant un parcours de formation les ayant amenées à des études 
supérieures, rapportent des formes d'inégalité et d'injustice liées à leur origine migratoire. Ce constat rejoint celui de Brinbaum, et al. (2015) : "Une fois prises en compte les caractéristiques sociales et familiales, les écarts entre les jeunes de la population majoritaire et les descendants d'immigrés se réduisent considérablement, mais ils persistent malgré tout $[\ldots] »($ p. 178).

\subsection{Des observateurs/trices privilégié-es}

Nous avons demandé aux futur-es enseignant-es s'ils ou elles avaient noté l'énonciation de stéréotypes ou de préjugés, ou observé des manifestations de discrimination des élèves issues de la migration dans le cadre de leurs stages ou éventuels remplacements. Notre présupposé était qu'elles/ils constituent des témoins privilégié-es de ces manifestations de par leur propre histoire migratoire, et qu'elles/ils se montrent particulièrement sensibles à ces phénomènes. L'ensemble des 25 personnes interrogées a rapporté au moins un élément signifiant. Les témoignages ne font presque jamais allusion à des actes délibérés ou à des paroles directement adressées aux élèves, mais portent plutôt sur des propos entendus en salle des maîtres et qui véhiculent certains stéréotypes ou préjugés susceptibles de conduire à des attentes moins élevées et de générer des prophéties autoréalisatrices (Jussim, 1986 ; Légal \& Delouvée, 2015) : "Tu verras, cet élève, il a beaucoup de difficultés, puis sa mère elle est arabe et elle ne sait pas parler français. Donc tu verras, il va redoubler cette année » $(\mathrm{V}$., 24 ans).

Parfois, ce sont des propos directement adressés par une enseignante à la personne en formation, ici une future enseignante justement ressortissante des Balkans : " J'ai l'impression que les élèves qui viennent des Balkans n'ont pas beaucoup de culture » (E., 28 ans).

Souvent, même lorsque ces propos sont clairement discriminatoires ou empreints de stéréotypes et de préjugés, les personnes interrogées disent en être choquées, mais avoir l'impression que les enseignant-es qui les profèrent n'en ont pas vraiment conscience. Ces constats rejoignent les éléments présents dans la littérature concernant la prise en compte de la diversité culturelle par des enseignant-es non issu-es de la migration. À ce sujet par exemple, Allemann-Ghionda (2001) affirme que :

Dans les systèmes scolaires examinés, il existe une contradiction fréquente entre les discours rhétoriques d'intégration et d'éducation interculturelle (dont aucun système scolaire ne fait désormais l'économie, car il faut être « up to date » et présentables au niveau international) et les pratiques scolaires. (p. 294)

Les attitudes dont il est question envers les élèves issu-es de la migration et leur famille sont caractérisées par l'ethnocentrisme (Ogay \& Edelmann, 2011) et le manque de décentration, davantage que par une volonté délibérée. Ces constats rejoignent aussi ceux de Bonnéry 
(2009) lorsqu'il met en évidence une attitude des enseignant-es oscillant entre l'indifférence aux différences et la surattention aux différences. Comme le rapporte cette étudiante :

En stage, je l'ai vraiment vécu, l'année passée. L'enseignante pensait faire du bien en se disant qu'elle enseignait dans un quartier populaire, qu'elle arriverait un peu à leur amener une certaine sérénité parce qu'elle était aussi dans la médiation et ce genre de choses. Mais j'ai l'impression qu'elle acceptait juste le fait que ce ne soit pas très grave s'ils n'avaient pas non plus le niveau attendu. Donc il y avait un problème à ce niveau-là je trouvais. [...] Elle pensait être bienveillante, mais finalement, par sa bienveillance, elle arrivait quand même à avoir des attitudes pas correctes. (B., 28 ans)

D'autres témoignages font également état de l'attribution a priori des difficultés de certains élèves au fait qu'elles/ils sont issu-es de la migration et du risque lié de réduction des attentes et de ne pas chercher d'autres explications (Moser, 2002).

\subsection{Des pistes pour favoriser les réussites scolaires et éducatives}

Nous avons également interrogé les futur-es enseignant-es issu-es de la migration sur ce qui serait à même de favoriser la réussite scolaire des élèves issu-es de la migration et de réduire les inégalités.

Les réponses s'orientent vers deux pôles : les parents et les élèves elles/eux-mêmes (les propositions visant les parents étant plus nombreuses), et impliquent l'enseignant-e dans ses actes et ses attitudes. Les pistes obtenues sont en cohérence avec les inégalités et discriminations subies ou observées mentionnées précédemment. Elles rejoignent également les constats de la littérature concernant une attention particulière à créer des ponts avec les familles issues de la migration (Changkakoti \& Broyon, 2013 ; Magaldi, et al., 2018).

L'accessibilité des informations - qu'elles soient à destination des élèves ou des parents concernant l'organisation de l'école et du système éducatif, les attentes, les enjeux liés à l'orientation dans les différentes voies possibles de scolarisation régulière ou vers l'enseignement spécialisé est une préoccupation souvent évoquée. La première raison évoquée est en lien avec l'allophonie, mais souvent les propositions dépassent la considération de la langue pour prendre en compte des différences de compréhension liées à la culture scolaire à laquelle les familles ont été confrontées préalablement. Cette attention dénote d'une conscience plus importante que chez les enseignant-es « majoritaires » (Moser, 2002 ; Suter, et al., 2009), de la présence de pratiques ethnocentrées (Ogay \& Edelmann, 2011).

La participation parentale est également une condition souvent évoquée pour les réussites scolaire et éducative des élèves issu-es de la migration. Deux formes de participation apparaissent majoritairement : l'accompagnement de la scolarité, par exemple l'aide pour les 
devoirs, l'intérêt manifesté pour le travail réalisé, et le fait de répondre aux invitations de l'école, par exemple à des séances d'information, mais également à d'autres occasions.

Plusieurs étudiant-es interrogent la distance entre l'école et certains parents, évoquant une double tendance : celle de la mise à l'écart des parents par les enseignant-es, et celle d'une distance des parents par rapport à l'école, liée à un sentiment d'incompétence :

Je pense qu'impliquer les parents, c'est très important parce que ce sont des parents qui sont aussi, je pense, un peu mis à l'écart. Et puis eux, ils ont aussi un peu une certaine gêne, je pense, vis-à-vis de l'enseignant, de ne pas pouvoir faire ou de ne pas pouvoir aider. (S., 25 ans)

En direction plus spécifique des élèves et concernant des aménagements pédagogiques, donc impliquant l'enseignement, ce sont les difficultés liées à l'apprentissage de la langue d'enseignement qui sont le plus souvent citées. Le plus souvent, les propositions vont dans le sens de mesures supplémentaires visant à réduire les difficultés passagères rencontrées pour cette raison. Une aide extérieure pour les devoirs semble constituer également une mesure intéressante aux yeux des personnes interrogées.

À de plus rares occasions, les pistes évoquées sont celles de la différenciation pédagogique, mais aussi de la prise en compte et de la valorisation de la langue et culture d'origine de l'élève, ainsi que de la valorisation des efforts fournis par ces élèves pour s'intégrer dans de nouvelles langue et culture. L'empathie, l'intérêt pour l'élève, son histoire, son vécu, reviennent également quelquefois spontanément dans les propositions des étudiant-es.

De manière générale, nous constatons que nous avons davantage recueilli des points d'attention et des axes d'intervention envisagés que des propositions concrètes d'actions. Nous mettons ceci en lien avec le fait que nous interrogeons de futur-es enseignant-es qui n'ont encore que peu d'expérience pratique.

\section{DISCUSSION ET CONCLUSION}

Les témoignages recueillis portant sur le vécu des personnes interrogées mettent en évidence des formes d'inégalités scolaires semblant d'abord basées sur des stéréotypes et préjugés, et portant sur la sous-évaluation des compétences et des propositions d'orientation privilégiant des filières à exigences élémentaires. Ces stéréotypes et préjugés agissent au moins à trois niveaux : celui de l'explication a priori des difficultés rencontrées lorsqu'il y en a ou de l'anticipation de difficultés supposées à venir, celui des attentes en termes de performances et de réussite scolaires, et enfin celui de l'orientation scolaire en fin de scolarité primaire. 
L'égalité des chances telle que définie par Dubet (2009), ou le critère d'équité caractérisant la justice sociale (Forsé, 2014), sont mis à mal dès lors que des différences de compétences liées au vécu migratoire sont présupposées. Ce n'est en effet pas l'investissement des individus qui est considéré dans une perspective méritocratique, mais leur performance à un moment donné, sans interroger ce qui a pu, de manière objective ou subjective, influencer ces performances.

Ce constat est également fait par les futur-es enseignant-es issu-es de la migration dans le cadre de leurs stages. Les discriminations observées concernent des attentes réduites quant aux performances scolaires d'élèves issu-es de la migration, le plus souvent en lien avec leur entourage familial et la langue parlée à la maison, et leurs conséquences sous forme de performances réduites, si l'on considère le rôle des prophéties autoréalisatrices (Légal \& Delouvée, 2015).

L'égalité d'accès ne semble a priori pas concernée dans notre système éducatif, si on la définit, à l'instar de Dubet (2009) comme accès à l'éducation élémentaire. Si l'on considère cependant spécifiquement l'orientation dans les différentes filières secondaires qui s'opère en fin de scolarité primaire et qui constituent les trois dernières années de la scolarité obligatoire, on observe bien, au travers de la sous-représentation des élèves issu-es de la migration dans les filières à exigences élevées, une forme « reportée » d'inégalité d'accès.

Les propositions visant les réussites scolaires et éducatives, tant dirigées vers les parents que vers les élèves, visent à promouvoir davantage de justice et peuvent principalement être rapprochées du critère d'égalité mis en évidence par Forsé (2014). Du point de vue des futures enseignant-es, il est pertinent de réduire les inégalités par des actions spécifiques visant une meilleure information et une compensation des désavantages temporaires liés aux lacunes dans la langue d'enseignement. Le rapprochement entre parents issus de la migration et l'école semble aussi constituer une piste privilégiée. Les propositions se rapprochent des principes de l'égalité des résultats avec la recherche d'une diminution des écarts entre les élèves issu-es de la migration et les autres.

Pour une partie des familles issues de la migration, nous constatons que, contrairement à la population majoritaire, l'accès au système éducatif revêt encore souvent des enjeux et des objectifs de mobilité sociale. Concernant leurs enfants, les injustices constatées au niveau de la sélection viennent contredire la perception d'un système éducatif dans lequel l'égalité d'accès est garantie.

Si l'on se réfère à la hiérarchisation des critères de justice sociale citée plus haut, nous constatons que, si le critère plébiscité par le système éducatif est celui de la reconnaissance des mérites, c'est justement ce critère qui est associé à la production des inégalités 
constatées. Les propositions des futur-es enseignant-es se situent quant à elles plutôt en lien avec le troisième niveau de la hiérarchie : la recherche d'une stricte égalité.

Pour reprendre l'idée de Fraser (2005), les « remèdes » proposés sont donc davantage correctifs que transformateurs. Provenant de futur-es enseignant-es primaires issu-es de la migration, elles-mêmes et eux-mêmes confronté-es à la diversité culturelle dans le cadre de leurs stages et témoins de formes d'injustices, ce constat nous semble cohérent. Les remèdes correctifs semblent en effet plus pragmatiques et applicables à l'échelle de l'enseignant-e. Des idées transformatrices - qui feront ultérieurement l'objet d'entretiens complémentaires quant à leur mise en œuvre - émergent cependant également dans leur discours. À ce stade, il s'agit davantage d'affirmation d'idéaux sur les valeurs qu'elles/ils souhaitent défendre ou qui devraient caractériser le système éducatif. Ce constat rejoint et exemplifie également le hiatus constaté par Allemann-Ghionda (2001) entre les discours officiels des politiques éducatives et les discours factuels.

Les étudiant-es issu-es de la migration interrogé-es se montrent par ailleurs assez critiques face aux discours rhétoriques. Elles/ils mettent en évidence leurs paradoxes et leur déficit d'application sur le terrain par des enseignant-es « majoritaires », pour certain-es peu sensibilisé-es aux questions interculturelles ou démuni-es face aux défis posés. La suite de la recherche devrait permettre d'établir si elles/ils y parviennent davantage ou mieux.

\section{RÉFÉRENCES}

Allemann-Ghionda, C. (2001). Les systèmes scolaires entre la rhétorique de l'intégration et la tentation de la séparation: Réflexions issues d'une recherche comparative. In C. Perregaux, T. Ogay, Y. Leanza, \& P. Dasen (Eds.), Intégrations et migrations. Regards pluridisciplinaires (pp. 269-302). Paris : L'Harmattan.

Allemann-Ghionda, C., Auernheimer, G., Grabbe, H., \& Krämer, A. (2006). Beobachtung und Beurteilung in soziokulturell und sprachlich heterogenen Klassen: Die Kompetenzen der Lehrpersonen. Zeitschrift für Pädagogik, 52(51), 250-266.

Bauer, S., \& Akkari, A. (2016). Les enseignants issus de la diversité ethnoculturelle représentent-ils une valeur ajoutée pour la profession ? Résultats d'une étude menée en Suisse romande. Canadian Journal of Education, 39(4), 1-25.

Bonnéry, S. (2009). Contenus, pratiques pédagogiques et échec scolaire. In M. Duru-Bellat, \& A. Van Zanten (Eds.), Sociologie du système éducatif (pp. 149-166). Paris : PUF.

Brinbaum, Y., Moguerou, L., \& Primon, J.-L. (2012). Les enfants d'immigrés ont des parcours scolaires différenciés selon leur origine migratoire. Insee Références Immigrés et descendants d'immigrés en France (pp. 43-49). Montrouge : INSEE.

Brinbaum, Y., Moguérou, L., \& Primon, J.-L. (2015). Les trajectoires du primaire au supérieur des descendants d'immigrés et de natifs d'un DOM. In C. Beauchemin, C. Hamel, \& P. 
Simon (Eds.), Trajectoires et origines. Enquête sur la diversité des populations en France (pp. 175-202). Paris : Ined éditions.

Changkakoti, N., \& Broyon, M.-A. (2013). Enseignants venus d'ailleurs : Tensions entre culture professionnelle et personnelle. Le cas de la Suisse romande. Revue internationale d'éducation de Sèvres, 63, 99-110.

Charles, F., \& Legendre, F. (2006). Les enseignants issus des immigrations : Modalités d'accès au groupe professionnel, représentations du métier et de l'école : Étude comparative. Paris : Sudel.

Conférence suisse des directeurs cantonaux de l'instruction publique (Ed.). (2003). Le parcours scolaire et de formation des élèves immigrés à "faibles » performances scolaires. Convegno 2002 : rapport final. Berne : CDIP.

Coradi Vellacott, M., \& Wolter, S. C. (2002). Origine sociale et égalité des chances. In Office fédéral de la statistique \& Conférence suisse des directeurs cantonaux de l'instruction publique (Eds.), Préparés pour la vie ? Les compétences de base des jeunes-Rapport national de l'enquête PISA 2000 (pp. 88-109). Neuchâtel : OFS \& CDIP.

Dhume, F. (2016). Du racisme institutionnel à la discrimination systémique ? Reformuler l'approche critique. Migrations Société, 1, 33-46.

Dubet, F. (2009). Penser les inégalités scolaires. In M. Duru-Bellat, \& A. Van Zanten (Eds.), Sociologie du système éducatif (pp. 17-34). Paris : PUF.

Forsé, M. (2014). Une comparaison internationale à propos des principes de justice sociale. In F. Dubet (Ed.), Inégalités et justice sociale (pp. 108-129). Paris : La Découverte.

Fraser, N. (2005). Qu'est-ce que la justice sociale ? Reconnaissance et redistribution (trad. E. Ferrarese). Paris : La Découverte.

Gomolla, M., \& Radtke, F.-O. (2009). Institutionelle Diskriminierung (3e éd.). Wiesbaden: VS Verlag für Sozialwissenschaften.

Jussim, L. (1986). Self-fulfilling prophecies : A theoretical and integrative review. Psychological review, 93(4), 429-445.

Karsenti, T., \& Savoie-Zajc, L. (2011). La recherche en éducation : Étapes et approches (3e éd.). Saint-Laurent : Éditions du renouveau pédagogique.

Kaufmann, J.-C. (2011). L'entretien compréhensif (4e éd.). Paris : Armand Colin.

Kronig, W. (2003). Eléments d'interprétation du faible taux de réussite scolaire des enfants immigrés dans le degré primaire. In Conférence suisse des directeurs cantonaux de l'instruction publique (Ed.), Le parcours scolaire et de formation des élèves immigrés à «faibles » performances scolaires (pp. 24-33). Berne: CDIP

Kronig, W. (2007). Die systematische Zufälligkeit des Bildungserfolgs: Theoretische Erklärungen und empirische Untersuchungen zur Lernentwicklung und zur Leistungsbewertung in unterschiedlichen Schulklassen (Vol. 32). Bern: Haupt Verlag AG.

Légal, J.-B., \& Delouvée, S. (2015). Stéréotypes, préjugés et discriminations. Paris : Dunod.

Magaldi, D., Conway, T., \& Trub, L. (2018). "I am here for a reason" : Minority teachers bridging many divides in urban education. Race Ethnicity and Education, 21(3), 306-318. 
Meyer, T. (2009). On ne prête qu'aux riches : L'inégalité des chances devant le système de formation en Suisse. In C. Suter, S. Perrenoud, R. Levy, U. Kuhn, D. Joye, \& P. Gazareth (Eds.), Rapport social 2008 (pp. 60-81). Zurich : Seismo.

Moser, U. (2002). La diversité à l'école : Un défi et une chance. In Office fédéral de la statistique \& Conférence suisse des directeurs cantonaux de l'instruction publique (Eds.), Préparés pour la vie ? Les compétences de base des jeunes - Rapport national de l'enquête PISA 2000 (pp.110-131). Neuchâtel : OFS \& CDIP.

Ogay, T., \& Edelmann, D. (2011). Penser l'interculturalité : L'incontournable dialectique de la différence culturelle. In A. Lavanchy, A. Gajardo, \& F. Dervin (Eds.), Anthropologies de l'interculturalité, (pp. 47-71). Paris : L'Harmattan.

Savoie-Zajc, L. (2009). L'entrevue semi-dirigée. In B. Gauthier (Ed.), Recherche sociale : de la problématique à la collecte des données (5e éd., pp. 337-360). Québec : Presses de l'université du Québec.

Suter, C., Perrenoud, S., Levy, R., Kuhn, U., Joye, D., \& Gazareth, P. (2009). Rapport social 2008, la Suisse mesurée et comparée. Zurich : Seismo.

Van der Maren, J.-M. (1996). Méthodes de recherche pour l'éducation. Bruxelles : Presses de l'Université de Montréal et de Boeck.

Villegas, A. M., \& Irvine, J. J. (2010). Diversifying the teaching force: An examination of major arguments. The Urban Review, 42(3), 175-192.

Open Access Publications - Bibliothèque de I'Université de Genève

Creative Commons Licence 4.0

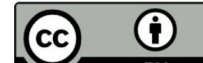

